\title{
The Central Role of Clinical Nutrition in COVID-19 Patients During and After Hospitalization in Intensive Care Unit
}

\author{
Francesco Ferrara ${ }^{1}$ (D) $\cdot$ Francesco De Rosa ${ }^{2} \cdot$ Antonio Vitiello $^{1}$ \\ Accepted: 10 July 2020 / Published online: 15 July 2020 \\ (C) Springer Nature Switzerland AG 2020
}

\begin{abstract}
The COVID-19-positive patient who is subject to a hyperinflammatory condition associated with lung injury with the development of pneumonia is hospitalized in the intensive care unit. Before resolving and overcoming the "cytokine storm," with overexpression of pro-inflammatory interleukins (IL-, Il-6), this patient will be intubated for more than $48 \mathrm{~h}$ and therefore needs adequate nutrition. Malnutrition can lead to sarcopenia with a decrease in lean body mass and worsening of the inflammatory state underway. In addition, severe debilitation, if not corrected with adequate nutrition, can greatly lengthen rehabilitation times with prolonged hospitalization, increased costs, and reduced turn over already in crisis due to the health emergency caused by coronavirus. The aim of this study is to focus attention on the nutritional importance that must be provided in case of COVID-19 together with pharmacological treatments to lower the number of circulating proinflammatory cytokines. Oral, enteral, and parenteral nutrition should always be carried out according to the patient's condition and, in the case of a hyperinflammatory patient, such as the one affected by COVID-19, it has been shown that the supplementation of amino acids helps to lower the inflammatory state and promotes normal physiological recovery.
\end{abstract}

Keywords Amino acids $\cdot$ COVID-19 $\cdot$ Nutrition $\cdot$ Glutamine $\cdot$ Hyperinflammatory $\cdot$ Sarcopenia

\section{Introduction}

A newly identified coronavirus (SARS-CoV-2) in China in December 2019 resulted in a significant number of pneumonia resulting in deaths. The spread, as usual of these viruses, is rapidly spreading all over the world with thousands of thousands of deaths. It is no coincidence that the WHO has declared a state of pandemic. For these reasons, worldwide attention is concentrated in the fight against this virus. The genomic sequence of SARS-CoV-2 is $79.5 \%$ of the identity with SARS-CoV. The evidence that caused Cov2 SARS infection causes a generalized hyperinflammatory state due to the release of cytokines "cytokine storm." In literature, it has been shown that the final stage of infection is the deadly one for humans. In fact, it is not the viral phase that kills but the strong

This article is part of the Topical Collection on COVID-19

Francesco Ferrara

francesco.ferrara@uslumbria1.it

1 Us1 Umbria 1, Pharmaceutical Department, Perugia, Italy

2 Campania Region Naples, Italy activation of the immune system and the consequent release of cytokines. The patient is thus supported and hospitalized in an intensive care unit (ICU) hoping that he will overcome the "famous storm" and remain alive. World scientific societies, health professionals, doctors, researchers, and scholars are intensifying research to find a suitable cure to fight the virus, both to prevent infection and to treat the patient before he arrives at death. The development of a vaccine will not be achieved in the short term. Many drugs are currently being tested to eradicate or at least reduce the lethality of the virus. Combinations of anti-inflammatory and antiviral drugs appear to have shown good efficacy in inhibiting the release of cytokines, thus preventing consequent lung collapse and also resulting in a drastic reduction in SARS-Cov-2-related mortality. The spontaneous reflection is the possibility of resorting to clinical nutrition and amino acid supplementation both to prevent, support, and overcome the infectious phase with greater ease also in combination with pharmacological treatments. This idea is reinforced by the fact that clinical nutrition and amino acids, in addition to adequately nourishing the patient bedridden in ICU, can modulate the immune system to prevent infection and ensure that the inflammatory state is kept under control with less release of cytokines. Above all, 
amino acids seem fundamental as an additional contribution in the patient suffering from COVID and hospitalized in ICU: on the one hand, it mitigates the release of cytokines and on the other, it favors a prompt de-hospitalization in the healing phase for a prompt turn-over in the pandemic phase and with consequent savings of indirect and intangible costs $[1,2]$.

\section{Methods}

COVID-19 disease leads to serious respiratory consequences such as pneumonia, respiratory failure, acute respiratory distress syndrome (ARDS), sepsis, and septic shock, which in many cases leads to urgent hospitalization in intensive care. ARDS is a severe hypoxic respiratory failure, edema, and risk of multi-organ dysfunction with invasive mechanical ventilation. Generally, patients who have died from SARS-CoV-2 infection are older and have 3 or more comorbidities. This is why COVID-19 is the new $\beta$-coronavirus, which can lead to various pulmonary phenomena and death and represents a global health challenge. Medical nutrition therapy is among the pillars of therapeutic principles and a fundamental tool among global therapeutic measures for patients with COVID-19. Malnourished people with low immunity and chronic diseases have a worse higher prognosis and mortality rates. The relevance of nutritional therapy is important and should be implemented as a first-line treatment and implemented in the anti-COVID-19 clinical practice standard. Good nutrition not only provides the body with immunity to disease, including COVID-19, but it is also the main guarantee for the promotion of the recovery of the disease and the infected patient. However, the indications for nutritional therapy must be tailored. Nutritionists should pay attention to the most appropriate artificial nutrition (AN) approach with enteral nutrition $(\mathrm{EN})$ or parenteral nutrition $(\mathrm{PN})$. Plan, the method, route, and formula of nutritional therapy must be adapted dynamically and promptly in accordance with the clinical characteristics of the COVID-19 patient. The transition between ONS, EN, and PN should be fluid, following the principle that when the EN can satisfy $50 \%$ of the target demand, the PN can be constantly reduced and carried out arrested; when the ONS can satisfy $50 \%$ of the target demand, the EN can be progressively reduced and therefore arrested [3, 4] (Fig. 1).

Old age and chronic diseases are related to frailty, a situation that leads to reduced functional reserves and reduced resistance to stress. Thus, there is weight loss, malnutrition, and acute hospitalization; if you do not intervene with adequate nutritional support, it can make an already compromised situation worse. All patients hospitalized for more than $48 \mathrm{~h}$, regardless of the initial body mass index (BMI) and age, are at risk of malnutrition and need adequate and timely nutrition. Prolonged hospitalization following catabolism and immobilization induces a reduction in weight and muscle mass which can cause sarcopenia, a condition which in turn helps to reduce respiratory and cardiac function, prolong the patient's hospitalization and a worse prognosis. In order to prevent malnutrition, the actions done by the hospital are improving the patient's response to therapy and facilitating healthcare professionals in the hospital nutrition management by reducing the already high workload due to the state of emergency. Most patients have severe inflammation and anorexia which leads to one drastic reduction of food intake which then leads to a respiratory failure treated with continuous positive airway pressure. High-calorie diets must be readily provided. Oral integration of whey protein and intravenous infusion of multivitamin and multimineral solutions must be implemented at the time of admission. If respiratory conditions worsen, total parenteral nutrition should be considered. To cope with the current emergency crisis, nutritional assistance must be implemented promptly and pragmatically in patients with COVID19 because it could be neglected although it is necessary for the benefit of clinical and effective results in preventing the consequences of malnutrition in this patient population $[5,6]$ (Fig. 2).

It is possible to follow simple patterns of conduct. In the critical patient in the intensive care with respiratory failure for over 48 h, EN medical nutritional therapy must be started with priority; PN nutrition has been started for more than 3 days after EN strategies have failed to avoid severe malnutrition. Overeating should always be avoided and a gradual approach is best. The nutritional requirement in COVID-19 must provide more energy than normal nutrition. Maintaining the energy balance of patients with COVID-19 is fundamental. However, considering the increased metabolic load in patients with severe pneumonia, moderately low calories can reduce the metabolic load and excessive energy intake is a risk factor. To reduce the catabolism due to inflammatory mediators, it is indicated to increase protein intake as a top priority. The supply of amino acids helps prevent muscle loss and improve the strength of the respiratory muscles. Given the importance of clinical, ethical, and economic action aimed at reducing infections, including those from COVID-19, studies have always been carried out which demonstrate how the correct supply of nutrients can improve the prognosis and pathological course in ICUs. The use of amino acids undoubtedly improves the clinical course and favors the prompt physiological recovery after a period of hospitalization. Then, in the case of the COVID patient, admitted to ICU with a strong inflammatory state, it is shown how the adequate nutritional support of glutamine, probiotics, and lactoferrin helps to reduce the proinflammatory molecules (IL-1, IL-6) responsible for the fatal event pulmonary coronavirus. Alterations in muscle protein turnover, if the patient remains hospitalized for a long time, could lead to physical disability. Hypercatabolic activity if integrated with amino acids could limit muscle hypercatabatabism $(\mathrm{MH})[7,8]$. 


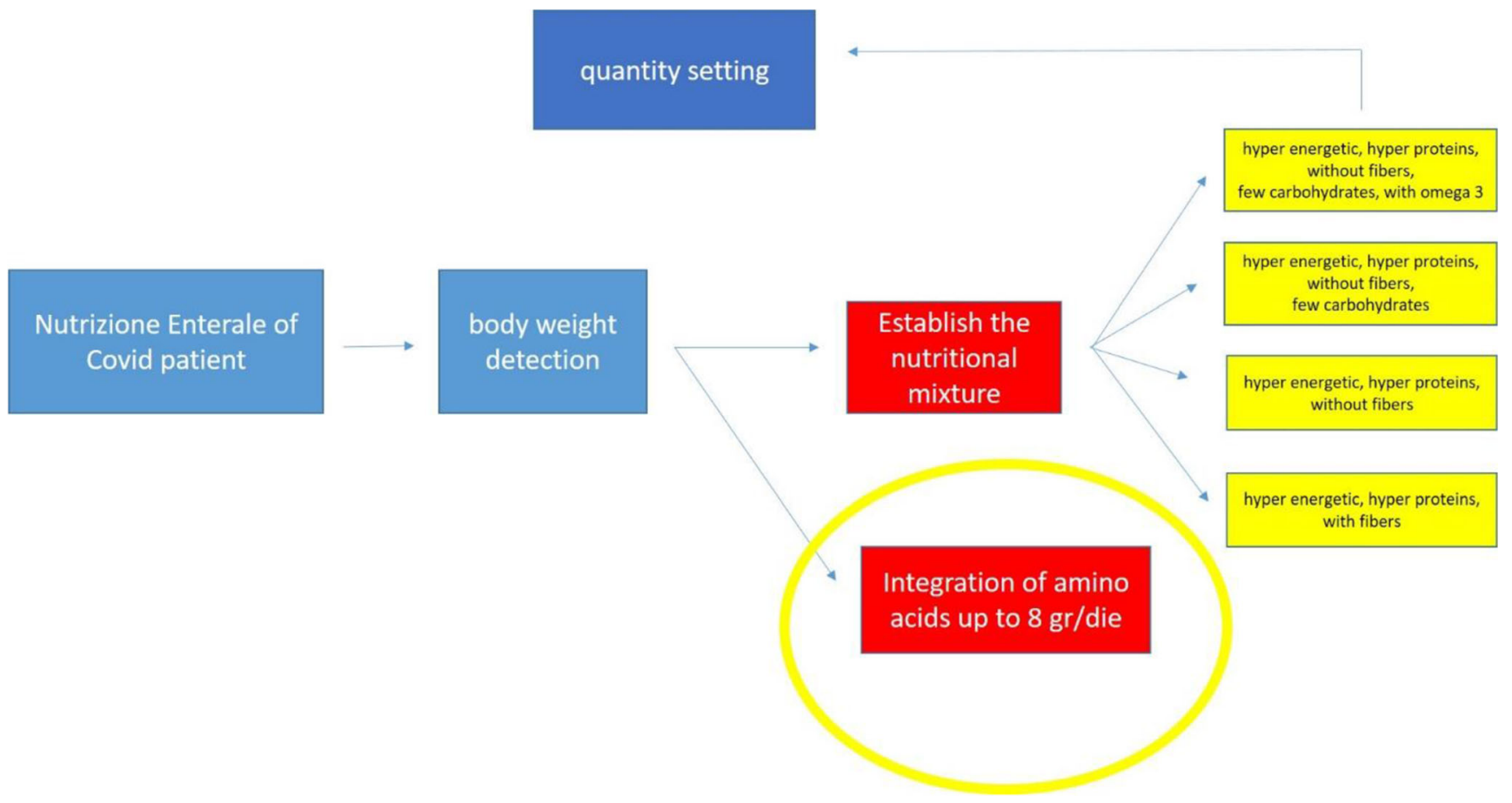

Fig. 1 Enteral nutritional support in the positive COVID patient

\section{Results and Discussion}

Inflammation and changes in amino acid metabolism are related to COVID-19 and affect the skeletal muscles; therefore, probably rehabilitation requires personalized approaches. In addition, amino acids are essential nutrients for infectious microorganisms and the levels of some amino acids such as tryptophan decrease in response to infection and/or inflammation, and for this reason, they should be restored so as not to experience severe malnutrition. Some nutrients, including amino acids, have been shown to have a remarkable amount of influence on immune function (immunonutrients) and improvement of metabolic and nutritional indices, such as nitrogen balance and serum proteins. Immunonutrients can promote patient recovery by inhibiting inflammatory responses and regulating immune function. Glutamine, which can modulate and preserve intestinal and lung function, is compromised in conditions of severe stress, reducing the production of pro-inflammatory cytokines [9, 10]. Cytokinemediated effects are an essential part response to infection but excessive production of pro-inflammatory cytokines increases the risk of pneumonia and death in COVID-19 patients. The production of pro-inflammatory cytokines (TH1 cytokines: e.g., tumor necrosis factor- $\alpha$ [TNF- $\alpha]$, IL-1, IL-6,
Fig. 2 Oral, enteral, or parenteral nutrition depending on clinical conditions
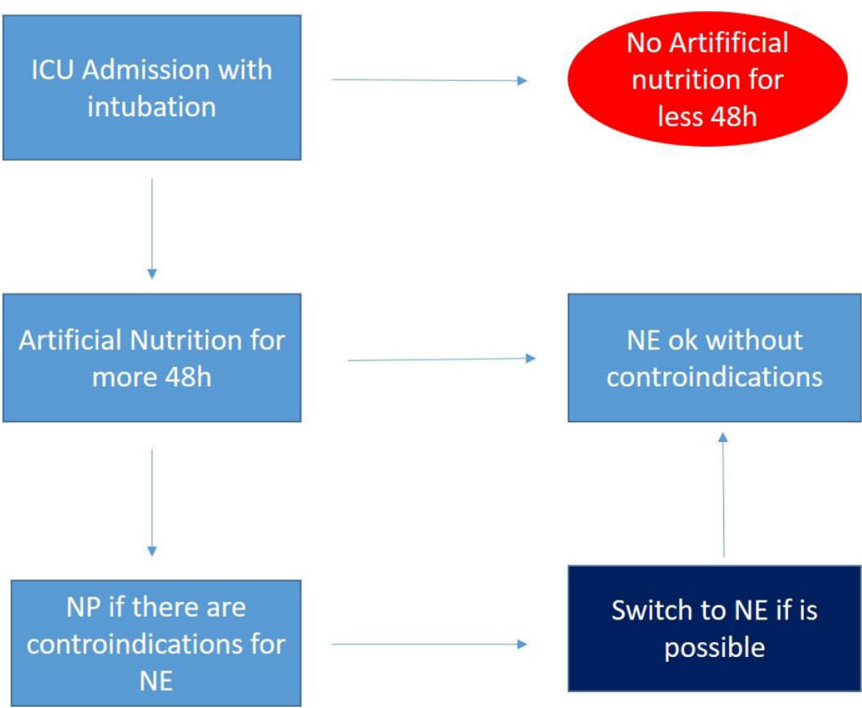

NE ok without 
interferon- $\gamma[$ IFN- $\gamma]$ ) in coronavirus pneumonia is overexpressed in a storm. Chronic inflammation occurs when the triggering factors persist or there is some sort of resolution failure processes. Inflammation plays a fundamental role in the pathogenesis of COVID-19: an imbalance between proinflammatory and anti-inflammatory cytokines, leading to the cytokine storm is currently considered to contribute to the development and progression of COVID-19. Many authors have shown that amino acids have anti-inflammatory effects and the integration of arginine, glutamine, or glycine improves lung damage induced by infections or any other inflammatory event thus contributing to alveolar improvement in its integrity and function. In particular, the administration of glutamine has been shown to significantly reduce inflammatory cytokines without affecting other parameters [11, 12]. Arginine or glycine supplementation may be a new nutritional strategy to reduce the deleterious effects of bacterial infection on alveolar function. Proinflammatory cytokines (TNF $\alpha$, IL-1 $\beta$, and IL-6) and chemotactic cytokines (CXCL-1, MCP-1, and ICAM-1) induce apoptosis in the alveoli and cause a severe inflammatory state. The use of amino acids reduced the mRNA levels for TNF $\alpha$, IL- $1 \beta$, and IL- 6 and reduced inflammation. IL-6 regulates the accumulation of neutrophils and therefore contributes to lung damage. The administration of arginine or glycine leads to a reduction in the expression of IL- 6 and CXCL- 1 with less neutrophils in the alveolar epithelium, so as to have a regulatory effect on the lung. Arginine or glycine has a regulatory function as adjuvant nutrients in clinical therapy. The molecular mechanisms underlying this important modulating effect of arginine, glycine, and glutamine are still to be clarified. Glutamine inhibits the accumulation of immune cells and arginine, glycine, or glutamine have inhibited cell apoptosis in various studies, but the metabolism of amino acids and their metabolites differ from each other in the alveoli, thus leading to different responses. Furthermore, it should be added that malnutrition easily leads to a decrease in the lean body mass and can cause sarcopenia, a frequently encountered disease in the elderly with aggravation of geriatric pathologies. Current opinions consider malnutrition and possible sarcopenia as a consequence of multiple medical, behavioral, and environmental factors involving the nervous, hormonal, nutritional system, substitution of muscle proteins, proinflammatory conditions, and the load of reactive oxygen species inside the mitochondria. With the intake of amino acids, there is a better functional recovery of the muscles and a significant reduction in serum cancer necrosis factor (TNF) and a significant increase in both insulin-like growth factors without having significant adverse effects. These preliminary data indicate that dietary supplements with the oral amino acid mixture have significantly increased lean body mass in elderly subjects with and without sarcopenia $[13,14]$.

\section{Conclusion}

The suggestions for proper evidence-based nutritional therapy reported here are for patients with critical COVID-19 conditions and should be included in life-saving therapies. The supplementation of amino acids and lactoferrin should always be accompanied by drug therapies following an ICU hospitalization and its importance cannot be overlooked. Malnutrition phenomena weaken the patient already in poor condition and greatly lengthen recovery and recovery times. Furthermore, the lung damage caused by coronavirus is caused by an overexpression of proinflammatory molecules (cytokines) which, from literature data, has been shown to be lowered by an adequate integration of amino acids. Nutritional therapy together with pharmacological therapy undoubtedly helps the COVID patient to overcome the acute phase of the disease first and to shorten recovery times.

Author Contributions The manuscript was written entirely by the authors. All authors made an equal contribution in the development of the paper.

\section{Compliance with Ethical Standards}

There are no sensitive data and no patients were recruited for this study.

Conflict of Interest The authors declare that they have no conflict of interest.

\section{References}

1. Caccialanza R, Laviano A, Lobascio F, Montagna E, Bruno R, Ludovisi S, et al. Early nutritional supplementation in noncritically ill patients hospitalized for the 2019 novel coronavirus disease (COVID-19): rationale and feasibility of a shared pragmatic protocol. Nutrition. 2020;74:110835. https://doi.org/10.1016/j.nut. 2020.110835 .

2. Romano L, Bilotta F, Dauri M, Macheda S, Pujia A, de Santis GL, et al. Short report - medical nutrition therapy for critically ill patients with COVID-19. Eur Rev Med Pharmacol Sci. 2020;24: 4035-9. https://doi.org/10.26355/eurrev 20200420874.

3. Dietetics and Clinical Nutrition Laboratory - Department of Public Health, Experimental and Forensic Medicine. Nutritional indications for patients with COVID-19 Accessed 29 March 2020

4. Cascella M, Rajnik M, Cuomo A, et al. Features, evaluation and treatment coronavirus (COVID-19) [Updated 2020 May 18]. In: StatPearls [Internet]. Treasure Island (FL): StatPearls Publishing; 2020 Jan-. Available from: https://www.ncbi.nlm.nih.gov/books/ NBK554776/

5. Dushianthan A, Grocott MP, Postle AD, Cusack R. Acute respiratory distress syndrome and acute lung injury. Postgrad Med J. 2011;87(1031):612-22. https://doi.org/10.1136/pgmj.2011. 118398 .

6. Singer P, Blaser AR, Berger MM, Alhazzani W, Calder PC, Casaer $\mathrm{MP}$, et al. ESPEN guideline on clinical nutrition in the intensive care unit. Clin Nutr. 2019;38(1):48-79. https://doi.org/10.1016/j. clnu.2018.08.037. 
7. Jin YH, Cai L, Cheng ZS, et al. A rapid advice guideline for the diagnosis and treatment of 2019 novel coronavirus (2019-nCoV) infected pneumonia (standard version). Mil Med Res. 2020;7(1):4. Published 2020 Feb 6. https://doi.org/10.1186/s40779-020-0233-6

8. SINPE. Guidelines for hospital artificial nutrition - special part B. Artificial Nutrition in respiratory failure.

9. Aquilani R, Emilio B, Dossena M, Baiardi P, Testa A, Boschi F, et al. Correlation of deglutition in subacute ischemic stroke patients with peripheral blood adaptive immunity: essential amino acid improvement. Int J Immunopathol Pharmacol. 2015;28(4):576-83. https://doi.org/10.1177/0394632015608249.

10. Aquilani R, Boselli M, D'Antona G, et al. Unaffected arm muscle hypercatabolism in dysphagic subacute stroke patients: the effects of essential amino acid supplementation. Biomed Res Int. 2014;2014:964365. https://doi.org/10.1155/2014/964365.

11. Solerte SB, Gazzaruso C, Bonacasa R, Rondanelli M, Zamboni M, Basso C, et al. Nutritional supplements with oral amino acid mixtures increases whole-body lean mass and insulin sensitivity in elderly subjects with sarcopenia. Am J Cardiol. 2008;101(11A):69E77E. https://doi.org/10.1016/j.amjcard.2008.03.004.
12. Buondonno I, Sassi F, Carignano G, Dutto F, Ferreri C, Pili FG, et al. From mitochondria to healthy aging: the role of branchedchain amino acids treatment: MATeR a randomized study. Clin Nutr. 2020;39:2080-91. https://doi.org/10.1016/j.clnu.2019.10. 013.

13. Inoue S, Ikeda H. Differences in plasma amino acid levels in patients with and without bacterial infection during the early stage of acute exacerbation of COPD Int J Chron Obstruct Pulmon Dis. 2019;14:575-583. Published 2019 Mar 1. https://doi.org/10.2147/ COPD.S188422.

14. Ma X, Zhang Y, Jiang D, Yang Y, Wu G, Wu Z. Protective effects of functional amino acids on apoptosis, inflammatory response, and pulmonary fibrosis in lipopolysaccharide-challenged mice. J Agric Food Chem. 2019;67(17):4915-22. https://doi.org/10.1021/acs. jafc.9b00942.

Publisher's Note Springer Nature remains neutral with regard to jurisdictional claims in published maps and institutional affiliations. 